\title{
Association Between Weekend Catch-Up Sleep and Metabolic Syndrome with Sleep Restriction in Korean Adults: A Cross-Sectional Study Using KNHANES
}

This article was published in the following Dove Press journal:

Diabetes, Metabolic Syndrome and Obesity: Targets and Therapy

\author{
Soo Min Son (iD 1 \\ Eun-Ju Park (D) \\ Young Hye Cho (D) \\ Sang Yeoup Lee $\mathbb{D}^{\prime}$ \\ Jung-In Choi $\mathbb{D}^{\prime}$ \\ Young-In Lee' \\ Yun Jin Kim (1) ${ }^{2}$ \\ Jeong Gyu Lee ${ }^{2}$ \\ Yu Hyeon $\mathrm{Yi}\left(\mathbb{D}^{2}\right.$ \\ Young Jin $\mathrm{Tak}^{2}$ \\ Hye Rim Hwang $\mathbb{D}^{2}$ \\ Seung-Hun Lee ${ }^{2}$ \\ Ryuk Jun Kwon (1D) \\ Choongrak $\mathrm{Kim}^{3}$ \\ 'Family Medicine Clinic and Research \\ Institute of Convergence of Biomedical \\ Science and Technology, Pusan National \\ University Yangsan Hospital, Yangsan, \\ Gyeongsangnam-do 50612, South Korea; \\ ${ }^{2}$ Department of Family Medicine and \\ Biomedical Research Institute, Pusan \\ National University Hospital, Busan \\ 626-770, South Korea; ${ }^{3}$ Department of \\ Statistics Pusan National University, Busan \\ 609-735, South Korea
}

Correspondence: Eun-Ju Park

Medical of Family Medicine, Department of Internal Medicine, Pusan National University Yangsan Hospital, Yangsan 50612

Tel +82-55-360-2I40

$\mathrm{Fax}+82-55-360-2170$

Email everbluel24@hanmail.net
Background: Many researchers have identified that adequate sleep duration is linked to the quality of life and metabolic diseases. Nowadays, it is hard to take enough sleep, so weekend catch-up sleep (CUS) may be an alternative option in modern society. To our knowledge, no previous studies reported the association between weekend CUS and metabolic syndrome, especially in the Korean population.

Objective: We investigated the association between weekend CUS and the prevalence of metabolic syndrome in Korean adults ( $\geq 20$ years old) with less than 6 hours of average weekday sleep.

Patients and Methods: A total of 1,453 individuals were selected from the Korean National Health and Nutrition Examination Survey. Weekend CUS was divided into four categories: $\leq 0$ hour, $0-1$ hour, $1-2$ hours, and $\geq 2$ hours. Odds ratios (ORs) with 95\% confidence intervals (CIs) were derived by univariate and multivariate logistic regression analyses.

Results: Participants with weekend CUS $\geq 1$ hour had decreased risk of metabolic syndrome in univariate analysis (CUS 1-2 hours: OR: 0.413 , 95\% CI: $0.301-0.568$; CUS $\geq 2$ hours: OR: $0.382,95 \%$ CI $0.296-0.493$ ). Weekend CUS $1-2$ hours reduced the risk of metabolic syndrome in multivariate logistic regression analysis (OR: 0.552, 95\% CI: 0.369-0.823). Based on the age group analysis, weekend CUS $\geq 1$ hour reduced the metabolic syndrome among those aged 20-39 and 40-65 (20-39: CUS 1-2 hours OR: 0.248, 95\% CI: 0.078-0.783, CUS $\geq 2$ hours OR: $0.374,95 \%$ CI: $0.141-0.991$; 40-65: CUS $1-2$ hours OR: $0.507,95 \%$ CI $0.309-0.832$ CUS $\geq 2$ hours OR: $0.638,95 \%$ CI: $0.415-0.981$ ).

Conclusion: Weekend CUS was associated with a low risk of metabolic syndrome among Korean adults with sleep restriction.

Keywords: sleep recovery, sleep deprivation, metabolic syndrome, insulin sensitivity

\section{Introduction}

Sleep plays a fundamental role in human physiology, including effects on development, immune response, and disease processes. ${ }^{1}$ Many researchers have identified the consequences of sleep deprivation, as well as ways to attain sufficient sleep and improve overall health and well-being. According to previous studies, adequate sleep duration is linked to the quality of life, and, more specifically, to type 2 diabetes, ${ }^{2}$ hypertension, ${ }^{3}$ metabolic syndrome, ${ }^{4}$ and mortality. ${ }^{5}$ These studies showed that $7-8$ 
hours of sleep is adequate and associated with low rates of metabolic disease and mortality.

Metabolic syndrome refers to a cluster of risk factors associated with insulin resistance, and cardiovascular disease. ${ }^{6}$ Patients with metabolic syndrome often develop diabetes mellitus, stroke, and other cardiovascular diseases and have a high rate of mortality. ${ }^{7}$ To reduce the prevalence of metabolic syndrome, lifestyle modification and pharmacologic therapy are important. ${ }^{8}$ In addition to calorie restriction, physical activity, and self-monitoring of metabolic factors, ${ }^{8}$ adequate sleep is essential. ${ }^{9}$ Sleep timing, quality, and duration are all associated with the prevalence of metabolic syndrome. ${ }^{10}$

Sleep deprivation seems inevitable in modern society. ${ }^{11}$ To compensate for the lack of sleep, weekend catch-up sleep (CUS) may be useful. Weekend CUS is weekend sleep meant to compensate for the sleep debt accrued during the week. Some studies have shown that weekend CUS is associated with a low prevalence of hypertension ${ }^{12}$ and obesity ${ }^{13}$ and beneficial for patients with insulin resistance. ${ }^{14}$ However, some studies claim that there is no metabolic advantage, even with regard to insulin sensitivity. ${ }^{15}$

The purpose of this study was to show the association between weekend CUS and metabolic syndrome in Korean adults with sleep restriction during weekday.

\section{Patients and Methods}

\section{Participants}

The participants were included from the Korean National Health and Nutrition Examination Survey (KNHANES) VII-1. The KNHANES is a national survey on health and nutritional status operated by the Korea Centers for Disease Control and Prevention (KCDC) and provides data representing Koreans. The present study was approved by the Institutional Review Board of Pusan National University Yangsan Hospital (IRB No. 05-202050). Each year, 25 households in 192 areas were sampled using a random sample to survey about 10,000 household members over 1 year of age. Depending on the life cycle stages of the participants, screening for various diseases, health surveys, and nutrition surveys were conducted. The data were collected via questionnaires, as well as standardized physical examination and blood sample analysis.

The participants were adults over 20 years of age with sleep averages of less than 6 hours per week. Lack of sleep duration in adults was less than 6 hours according to the National Sleep Foundation in the US. ${ }^{16}$ Of the total 16,277 participants from KNHANES VII-1, we excluded patients diagnosed with any type of cancer, including gastric, hepatocellular, colon, breast, cervical, thyroid, and other cancers. Pregnant women, shift workers, and any participants for whom no data were collected were also excluded.

\section{Baseline Characteristics}

Based on questionnaire responses and examination, data were collected for age, sex, body mass index (BMI), smoking status, alcohol consumption status, physical activity, income, marital status, average sleep duration and social jetlag. Age was divided into three categories: 20-39, $40-65$ and $>65$ years. BMI is calculated as weight $(\mathrm{kg})$ divided by height $(\mathrm{m})$ squared. Smoking status was indicated as never smokers, ex-smokers, and current smokers. Alcohol consumption was defined as an average drinking frequency of at least once per month during the preceding year. According to KNHANES, regular physical activity was defined as at least 2 hours 30 minutes of moderate physical activity or 1 hour 15 minutes of high-intensity physical activity per week. We also included regular physical activity participants who mixed moderate-intensity physical activity with high-intensity physical activity (high intensity, 1 minute; medium intensity, 2 minutes) per week. Income was categorized according to the quartile of the KNHANES. We considered low-middle and high-middle as "middle income" status. Marital status was indicated as single or married. Although excluded shifting workers, we found that alterations in sleep time during workdays and free days affected sleep timing subsequent day. ${ }^{17}$ This misalignment between social and biological time can be described as "social jetlag."18 The relative social jetlag was calculated as mid-sleep time on free day (MSF) minus mid-sleep time on work day (MSW), and we used the absolute social jetlag to evaluate social jetlag. ${ }^{19}$ Each variable was calculated according to the following formula: $\mathrm{MSF}=$ sleep onset on free day $+($ sleep duration on free day $) / 2, \mathrm{MSW}=$ sleep onset on work day $+($ sleep duration on workday $) / 2 .{ }^{19}$

\section{Weekend CUS}

Weekend CUS values were used to obtain average weekday (or workday) sleep duration and average weekend (or free day) sleep duration values from response to self-report questionnaires. Weekend CUS was calculated as the average weekend sleep duration minus average weekday sleep duration, ${ }^{20}$ and this was divided into four categories: $\leq 0$ hour, $0-1$ hour, $1-2$ hours, and $\geq 2$ hours. Non-CUS has the same meaning as CUS $\leq 0$ hour. 


\section{Metabolic Syndrome}

Metabolic syndrome was defined, based on the modified NCEP ATP III following Asian standards for abdominal obesity $^{21}$ and metabolic syndrome, when more than three of the five criteria were met: 1) Abdominal obesity: waist circumference $\geq 90 \mathrm{~cm}$ in men and $\geq 85 \mathrm{~cm}$ in women; 2 ) High blood pressure: $\geq 130 / 85 \mathrm{mmHg}$ or treatment for hypertension; 3) Hypertriglyceridemia: triglyceride $\geq 150 \mathrm{mg} / \mathrm{dL}$ or treatment for dyslipidemia; 4) Low serum HDL level: $<40 \mathrm{mg} / \mathrm{dL}$ in men and $<50 \mathrm{mg} / \mathrm{dL}$ in women; 5) Impaired blood glucose: fasting plasma glucose $\geq 100 \mathrm{mg} / \mathrm{dL}$ or treatment for diabetes.

\section{Statistical Analyses}

Baseline characteristics, including basic demographics, physical measurements, and continuous variables, of the four CUS groups were compared using analysis of variance and presented as mean \pm standard deviation. To investigate the distribution of each group, categorical variables were compared using the chi-square test and presented as percentages. Univariate logistic regression analyses were performed to evaluate whether metabolic syndrome was associated with weekend CUS. To further evaluate the association between metabolic syndrome and weekend CUS, we performed multivariate logistic regression analyses, adjusted for age, sex, BMI, physical activity, income, marital status and social jetlag. To evaluate the association between metabolic syndrome and weekend
CUS by age group, we performed multivariate logistic regression analyses which were adjusted for sex, BMI, physical activity, income, marital status and social jetlag. IBM SPSS version 20 was used for data management and analysis. The level of statistical significance was set at $p<0.05$.

\section{Results \\ Participants}

At baseline, 1,453 participants were included out of a total of 16,277 individuals. The total number of people meeting the exclusion criteria was 14824 . The non-CUS group comprised 687 participants, and the weekend CUS groups comprised 766 participants (0-1 hour of CUS: $64 ; 1-2$ hours: 241; $\geq 2$ hours: 461; Figure 1).

\section{Baseline Characteristics}

Table 1 shows the baseline characteristics of the participants. Mean age was lower for individuals with weekend CUS than for non-CUS participants. Mean age was also lower for those with longer average weekend CUS duration ( $\leq 0$ hour: $60.78 \pm 14.33$ y, $0-1$ hour: $50.72 \pm 15.16$; $1-2$ hours: $46.91 \pm 13.96$; $\geq 2$ hours: $40.99 \pm 15.06$; $\mathrm{p}<0.05$ ). BMI, physical activity, income, and marital status were also significantly different between the groups. The nonCUS group had a shorter average sleep duration than the weekend CUS groups during the weekend ( $\leq 0$ hour: 4.72 \pm 0.83 ; 0-1 hour: $5.74 \pm 0.47$; $1-2$ hours: $6.38 \pm 0.64$; $\geq 2$

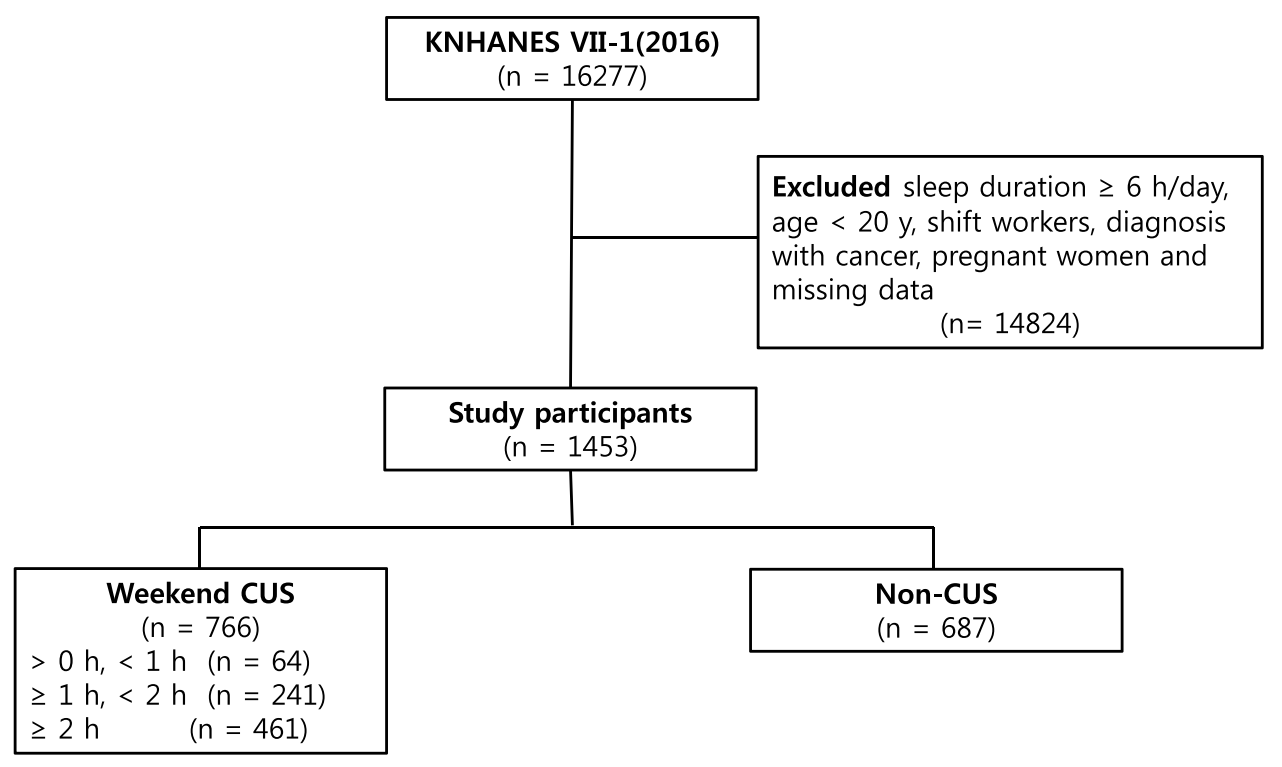

Figure I Flow diagram of study participants in KNHANES VII-I 2016.

Abbreviations: KNHANES, Korean National Health and Nutrition Examination Survey; CUS, catch-up sleep; h, hour (s); y, years old; n, number. 
Table I Baseline Characteristics of Study Participants

\begin{tabular}{|c|c|c|c|c|c|}
\hline & \multirow{2}{*}{$\begin{array}{l}\text { Non-CUS } \\
\leq 0\end{array}$} & \multicolumn{3}{|c|}{ Weekend CUS (h) } & \multirow[t]{2}{*}{$p$ value } \\
\hline & & $>0,<1$ & $\geq 1,<2$ & $\geq 2$ & \\
\hline Age $(y)$ & $60.78 \pm 14.33$ & $50.72 \pm 15.16$ & $46.91 \pm 13.96$ & $40.99 \pm 15.06$ & \multirow{4}{*}{$<0.05^{*}$} \\
\hline $20-39, n(\%)$ & $68(9.9)$ & $14(21.9)$ & $76(31.5)$ & $212(46.0)$ & \\
\hline $40-65$ & $329(47.9)$ & $38(59.4)$ & 142(58.9) & $220(47.7)$ & \\
\hline$>65$ & $290(42.2)$ & $12(18.8)$ & $23(9.5)$ & $29(6.3)$ & \\
\hline $\begin{array}{l}\text { Gender } \\
\text { Female, } n(\%)\end{array}$ & $419(61.0)$ & $40(62.5)$ & $143(59.3)$ & $274(59.4)$ & 0.918 \\
\hline BMI, (kg/m2) & $24.86 \pm 3.85$ & $24.74 \pm 3.57$ & $24.47 \pm 3.81$ & $23.7 I \pm 3.73$ & $<0.05 \dagger$ \\
\hline \multicolumn{6}{|l|}{ Smoking Status, $n(\%)$} \\
\hline Never & $423(61.6)$ & $40(62.5)$ & $157(65.1)$ & $292(63.3)$ & \multirow[t]{3}{*}{0.444} \\
\hline Ex & $133(19.4)$ & $9(14.1)$ & $43(17.8)$ & $70(15.2)$ & \\
\hline Current & $131(19.1)$ & $15(23.4)$ & $4 I(I 7.0)$ & $99(21.5)$ & \\
\hline \multicolumn{6}{|l|}{ Drinking ${ }^{\mathrm{a}}, \mathrm{n}(\%)$} \\
\hline Yes & $316(55.6)$ & $35(61.4)$ & $126(58.3)$ & $266(62.0)$ & 0.232 \\
\hline \multicolumn{6}{|l|}{ Physical Activity, n(\%) } \\
\hline Regular $^{\mathrm{b}}$ & $254(37.2)$ & $31(49.2)$ & $118(49.2)$ & $248(53.8)$ & $<0.05^{*}$ \\
\hline \multicolumn{6}{|l|}{ Income ${ }^{c}, n(\%)$} \\
\hline Low & $207(30.2)$ & $8(12.5)$ & $69(28.6)$ & $122(26.5)$ & $0.018 *$ \\
\hline Middle & $326(47.6)$ & $4 I(64 . I)$ & $103(42.7)$ & $229(49.7)$ & \\
\hline High & $152(22.2)$ & $15(23.4)$ & $69(28.6)$ & $110(23.9)$ & \\
\hline \multicolumn{6}{|l|}{ Marital Status, n(\%) } \\
\hline Married & $633(92.1)$ & $53(82.8)$ & $198(82.2)$ & $299(64.9)$ & $<0.05^{*}$ \\
\hline Average Sleep Duration, (h) & & & & & $<0.05^{\dagger}$ \\
\hline Weekday & $4.76 \pm 0.81$ & $5.26 \pm 0.48$ & $5.12 \pm 0.53$ & $4.83 \pm 0.76$ & \\
\hline Weekend & $4.72 \pm 0.83$ & $5.74 \pm 0.47$ & $6.38 \pm 0.64$ & $8.08 \pm 1.27$ & \\
\hline Social jetlag ${ }^{\mathrm{d}}$, (h) & $0.55 \pm 2.77$ & $0.79 \pm 0.78$ & $1.07 \pm 1.27$ & $2.00 \pm 2.33$ & $<0.05^{\dagger}$ \\
\hline \multicolumn{6}{|l|}{ Metabolic syndrome } \\
\hline$n(\%)$ & $335(48.8)$ & $25(39.1)$ & $68(28.2)$ & $123(26.7)$ & $<0.05^{*}$ \\
\hline
\end{tabular}

Notes: $p<0.05$, *Based on chi-square test. †Based on ANOVA. Values are presented as mean \pm standard deviation or number (percentage). ${ }^{\ddagger}$ Non-CUS means CUS $\leq$ 0 hour. ${ }^{a}$ Average drinking frequency of at least once per month during the preceding year. ${ }^{b}$ At least 2.5 hours of moderate activity or I.25 hours of high-intensity activity per week defined by KNHANES. 'Categorized according to quartile of the KNHANES; consider low-middle and high-middle as "middle" status. 'Social jetlag = I MSF $\mathrm{MSW} I ; \mathrm{MSF}=\mathrm{SOF}+(\mathrm{SDF}) / 2 ; \mathrm{MSW}=\mathrm{SOW}+(\mathrm{SDW}) / 2$.

Abbreviations: CUS, catch-up sleep; BMI, body mass index ( $\mathrm{kg} / \mathrm{m} 2)$; y, years old; $\mathrm{n}$, number; h, hour (s); ANOVA, analysis of variance; KNHANES, Korean National Health and Nutrition Examination Survey; MSF, mid-sleep time on free day; MSW, mid-sleep time on work day; SOF, sleep onset on free day; SDF, sleep duration on free day; SOW, sleep onset on work day; SDW, sleep duration on work day.

hours: $8.08 \pm 1.27)$. Mean CUS duration was $0.486 \pm 0.019$ for the group with CUS $0-1$ hour, $1.252 \pm 0.018$ for the group with CUS $1-2$ hours, and $3.294 \pm 0.070$ for those with CUS $\geq 2$ hours.

\section{Association Between Weekend CUS and Metabolic Syndrome}

Univariate logistic regression analyses were performed to determine whether there was a significant association between weekend CUS and metabolic syndrome. We used the non-CUS group as a reference. Participants with weekend CUS of more than 1 hour had a significantly decreased risk of metabolic syndrome (weekend CUS 1-2 hours odds ratio [OR]: $0.413,95 \%$ confidence interval [CI]: 0.301-0.568; weekend CUS $\geq 2$ hours OR: $0.382,95 \%$ CI: $0.296-0.493$; Table 2).

Multivariate logistic regression analyses were performed to evaluate the association between weekend CUS and metabolic syndrome, adjusted for age, sex, BMI, physical activity, income, marital status and social jetlag. There was a significant 
Table 2 Odds Ratios (95\% Confidence Intervals) for Metabolic Syndrome

\begin{tabular}{|l|l|l|}
\hline & Model I & Model 2 \\
\hline $\begin{array}{l}\text { Weekend CUS } \\
\leq 0 \text { hour }\end{array}$ & \\
$>0$ hour, $<1$ hour & $0.674(0.399-1.137)$ & Reference \\
$\geq$ I hour, <2 hours & $0.413(0.30 I-0.568)^{*}$ & $0.552(0.367-1.707)$ \\
$\geq 2$ hours & $0.382(0.296-0.493)^{*}$ & $0.809(0.577-1.135)$ \\
\hline
\end{tabular}

Notes: *p value $<0.05$, Model I, univariate logistic regression, unadjusted Model 2, multivariate logistic regression, adjusted for age, sex, BMI, physical activity, income, marital status and social jetlag.

Abbreviation: CUS, catch-up sleep.

decrease in metabolic syndrome for those with weekend CUS of 1-2 hours, compared with non-CUS participants (weekend CUS 1-2 hours OR: 0.552, 95\% CI: 0.369-0.823; Table 2). For those with weekend CUS $\geq 2$ hours, the prevalence of metabolic syndrome was low, but this was not statistically significant (weekend CUS $\geq 2$ hours OR: 0.809, 95\% CI: 0.577-1.135).

\section{Association Between Metabolic Syndrome and Weekend CUS by Age Group}

There was a significant decrease in metabolic syndrome for those with a weekend CUS $\geq 1$ hour, compared with individuals in the non-CUS group who were 20-39 or 40-65 years old (20-39: weekend CUS 1-2 hours OR: 0.248, 95\% CI: 0.078-0.783 weekend CUS $\geq 2$ hours OR: 0.374, 95\% CI: 0.141-0.991; 40-65: weekend CUS 1-2 hours OR: $0.507,95 \%$ CI $0.309-0.832$ weekend CUS $\geq 2$ hours OR: 0.638, 95\% CI: 0.415-0.981). However, there was a significant increase in the prevalence of metabolic syndrome for those with weekend CUS $\geq 2$ hours, compared with members of the non-CUS group who were $>65$ years old (weekend CUS $\geq 2$ hours OR: 4.143, 95\% CI: $1.606-10.689$, Table 3 ). The effect of age on the prevalence of metabolic syndrome by weekend CUS did not increase the risk of metabolic syndrome among those aged 20-39 and 40-65 years. On the other hand, the effect of age on the prevalence of metabolic syndrome by weekend CUS increased the risk of metabolic syndrome among those $>65$ years old.

\section{Discussion}

The results of our study suggest that weekend CUS compensates for the metabolic disadvantages caused by lack of sleep during the week. Specifically, weekend CUS is linked to a low prevalence of metabolic syndrome among Korean adults with less than 6 hours of sleep per day, and the prevalence of metabolic syndrome was significantly lower with CUS 1-2 hours. Even though the results of the multivariate logistic regression analysis did not indicate a significant difference, the results of univariate logistic regression analysis showed that more than 2 hours of weekend CUS was associated with a reduced risk of metabolic syndrome compared to participants with non-CUS. Based on the age group analysis, weekend CUS $\geq 1$ hour decreased the risk of metabolic syndrome among those aged 20-39 and 40-65 years.

Sleep recovery may reverse the mechanism by which sleep deprivation causes metabolic syndrome. Insulin resistance is one of the key factors of metabolic syndrome ${ }^{22}$ Killick et al reported that insulin sensitivity was higher after three nights of sleep extension than for sustained sleep restriction in healthy men who slept in the same sleep pattern for at least 6 months and, on average, 5 years. ${ }^{14}$ Appetite and the autonomic nervous system are also related to CUS and metabolism. Sleep restriction inhibits leptin secretion by sympathetic activation $^{23,24}$ and promotes ghrelin release prohibited by parasympathetic activity. ${ }^{25,26}$ These factors cause an increase in appetite and a decrease in satiety and, thereby, increase obesity. ${ }^{27}$ Spiegel reported that the leptin level was $19 \%$ lower in a sleep-restricted group after 6 days of 4-hour daily sleep duration than in participants who experienced sleep extension. ${ }^{28}$ Finally, weekend CUS may be involved

Table 3 Odds Ratios (95\% Confidence Intervals) for Metabolic Syndrome by Age Group

\begin{tabular}{|l|l|l|l|}
\hline & $20-39(y)$ & $40-65(y)$ & $>65(y)$ \\
\hline Weekend CUS & & & \\
$\leq 0$ hour & Reference & Reference & Reference \\
$>0$ hour, $<1$ hour & $0.426(0.069-2.63 I)$ & $0.7 I 2(0.304-1.668)$ & $1.53 I(0.40 I-5.84 I)$ \\
$\geq 1$ hour, $<2$ hours & $0.248(0.078-0.783)^{*}$ & $0.507(0.309-0.832)^{*}$ & $0.688(0.263-1.80 I)$ \\
$\geq 2$ hours & $0.374(0.14 I-0.99 I)^{*}$ & $0.638(0.4 I 5-0.98 I)^{*}$ & $4.143(I .606-10.689)^{*}$ \\
\hline
\end{tabular}

Notes: * $p$ value $<0.05$, multivariate logistic regression, adjusted for sex, BMI, physical activity, income, marital status and social jetlag. Abbreviations: $y$, years old; CUS, catch-up sleep. 
in the recovery of oxidative stress and inflammatory response. Oxidative stress is known to be involved in metabolic diseases, such as obesity, cardiovascular disease, and metabolic syndrome. ${ }^{29,30}$ Pihl et al $^{30}$ showed that waist circumference correlates with high-sensitivity C-reactive protein (hsCRP), suggesting that CRP is related to BMI and obesity. Recently, Jung suggested that weekend CUS may lower serum hsCRP levels and reduce the risk of cardiovascular disease among Korean workers. ${ }^{31}$ Similar to our study, they demonstrated that participants who had CUS of 1-2 hours on weekends had a significantly lower risk of hsCRP elevation than those with non-CUS.

In our study, mean age was lower for participants with weekend CUS and those with longer average weekend CUS duration. Dominguez and Barbagallo reported that aging contributed significantly to the increased prevalence of metabolic syndrome. ${ }^{32}$ Our study also showed that age lowered its prevalence in the weekend CUS group which included relatively younger participants. This effect appeared in participants $>65$ years old and significantly contributed to the prevalence of metabolic syndrome, rather than CUS.

Our study constraints limit our conclusions. We used a cross-sectional study design that hinders the identification of a causal association between weekend CUS and metabolic syndrome, and we had no information on sleep quality or nap or sleep disorders, including sleep apnea and insomnia. Furthermore, it was not possible to determine whether an individual's sleep pattern had been maintained long term or recently changed. Nonetheless, our study is valuable in its representation of the general population of Korea.

\section{Conclusion}

Our study shows that weekend CUS is associated with a low prevalence of metabolic syndrome among Korean adults with less than 6 hours of sleep per day. Maintaining adequate daily sleep duration is beneficial in protecting against metabolic disease. If adequate daily sleep is not possible, weekend CUS may be a practical alternative. Further studies are necessary to clarify the causal association between recovery sleep and metabolic syndrome.

\section{Disclosure}

The authors report no conflicts of interest in this work.

\section{References}

1. Zielinski MR, McKenna JT, McCarley RW. Functions and mechanisms of sleep. AIMS Neurosci. 2016;3:67-104. doi:10.3934/ Neuroscience.2016.1.67
2. Shan Z, Ma H, Xie M, et al. Sleep duration and risk of type 2 diabetes: a meta-analysis of prospective studies. Diabetes Care. 2015;38:529-537. doi:10.2337/dc14-2073

3. Gangwisch JE, Heymsfield SB, Boden-Albala B, et al. Short sleep duration as a risk factor for hypertension: analyses of the first National Health and Nutrition Examination Survey. Hypertension. 2006;47:833-839. doi:10.1161/01.HYP.0000217362.34748.e0

4. Hall MH, Muldoon MF, Jennings JR, Buysse DJ, Flory JD, Manuck SB. Self-reported sleep duration is associated with the metabolic syndrome in midlife adults. Sleep. 2008;31:635-643. doi:10. 1093/sleep/31.5.635

5. Cappuccio FP, D’Elia L, Strazzullo P, Miller MA. Sleep duration and all-cause mortality: a systematic review and meta-analysis of prospective studies. Sleep. 2010;33:585-592. doi:10.1093/sleep/33.5.585

6. Simmons RK, Alberti KG, Gale EA, et al. The metabolic syndrome: useful concept or clinical tool? Report of a WHO expert consultation. Diabetologia. 2010;53:600-605. doi:10.1007/s00125-009-1620-4

7. Obunai K, Jani S, Dangas GD. Cardiovascular morbidity and mortality of the metabolic syndrome. Med Clin North Am. 2007;91:116984, x. doi:10.1016/j.mena.2007.06.003

8. Takahara M, Shimomura I. Metabolic syndrome and lifestyle modification. Rev Endocr Metab Disord. 2014;15:317-327. doi:10. 1007/s11154-014-9294-8

9. Koren D, Dumin M, Gozal D. Role of sleep quality in the metabolic syndrome. Diabetes Metab Syndr Obes. 2016;9:281-310. doi:10. 2147/DMSO.S95120

10. Wu MC, Yang YC, Wu JS, Wang RH, Lu FH, Chang CJ. Short sleep duration associated with a higher prevalence of metabolic syndrome in an apparently healthy population. Prev Med. 2012;55:305-309. doi:10.1016/j.ypmed.2012.07.013

11. Knutson KL, Van Cauter E, Rathouz PJ, DeLeire T, Lauderdale DS. Trends in the prevalence of short sleepers in the USA: 1975-2006. Sleep. 2010;33:37-45. doi:10.1093/sleep/33.1.37

12. Hwangbo Y, Kim WJ, Chu MK, Yun CH, Yang KI. Association between weekend catch-up sleep duration and hypertension in Korean adults. Sleep Med. 2013;14:549-554. doi:10.1016/j.sleep.2013.02.009

13. Kim CW, Choi MK, Im HJ, et al. Weekend catch-up sleep is associated with decreased risk of being overweight among fifth-grade students with short sleep duration. J Sleep Res. 2012;21:546-551. doi:10.1111/j.1365-2869.2012.01013.x

14. Killick R, Hoyos CM, Melehan KL, Dungan GC 2nd, Poh J, Liu PY. Metabolic and hormonal effects of 'catch-up' sleep in men with chronic, repetitive, lifestyle-driven sleep restriction. Clin Endocrinol (Oxf). 2015;83:498-507. doi:10.1111/cen.12747

15. Depner CM, Melanson EL, Eckel RH, et al. Ad libitum weekend recovery sleep fails to prevent metabolic dysregulation during a repeating pattern of insufficient sleep and weekend recovery sleep. Curr Biol. 2019;29:957-67 e4. doi:10.1016/j.cub.2019.01.069

16. Hirshkowitz M, Whiton K, Albert SM, et al. National sleep foundation's updated sleep duration recommendations: final report. Sleep Health. 2015;1:233-243. doi:10.1016/j.sleh.2015.10.004

17. Zerbini G, Kantermann T, Merrow M. Strategies to decrease social jetlag: reducing evening blue light advances sleep and melatonin. Eur J Neurosci. 2018. doi:10.1111/ejn.14293

18. Wittmann M, Dinich J, Merrow M, Roenneberg T. Social jetlag: misalignment of biological and social time. Chronobiol Int. 2006;23:497-509. doi:10.1080/07420520500545979

19. Roenneberg T, Allebrandt KV, Merrow M, Vetter C. Social jetlag and obesity. Curr Biol. 2012;22:939-943. doi:10.1016/j.cub.2012.03.038

20. Oh YH, Kim H, Kong M, Oh B, Moon JH. Association between weekend catch-up sleep and health-related quality of life of Korean adults. Medicine (Baltimore). 2019;98:e14966. doi:10.1097/MD.000 0000000014966

21. Nam GE, Kim SM, Han K, et al. Metabolic syndrome and risk of Parkinson disease: a nationwide cohort study. PLoS Med. 2018;15: e1002640. doi:10.1371/journal.pmed.1002640 
22. Roberts CK, Hevener AL, Barnard RJ. Metabolic syndrome and insulin resistance: underlying causes and modification by exercise training. Compr Physiol. 2013;3:1-58. doi:10.1002/cphy.c110062

23. Rayner DV, Trayhurn P. Regulation of leptin production: sympathetic nervous system interactions. J Mol Med (Berl). 2001;79:8-20. doi:10.1007/s001090100198

24. Kiba T. Relationships between the autonomic nervous system and the pancreas including regulation of regeneration and apoptosis: recent developments. Pancreas. 2004;29:e51-8. doi:10.1097/00006676200408000-00019

25. van der Lely AJ, Tschop M, Heiman ML, Ghigo E. Biological, physiological, pathophysiological, and pharmacological aspects of ghrelin. Endocr Rev. 2004;25:426-457. doi:10.1210/er.2002-0029

26. Sugino T, Yamaura J, Yamagishi M, et al. Involvement of cholinergic neurons in the regulation of the ghrelin secretory response to feeding in sheep. Biochem Biophys Res Commun. 2003;304:308-312. doi:10.1016/S0006-291X(03)00593-X

27. Morselli L, Leproult R, Balbo M, Spiegel K. Role of sleep duration in the regulation of glucose metabolism and appetite. Best Pract Res Clin Endocrinol Metab. 2010;24:687-702. doi:10.1016/j.beem.2010. 07.005
28. Spiegel K, Leproult R, L'Hermite-Baleriaux M, Copinschi G, Penev PD, Van Cauter E. Leptin levels are dependent on sleep duration: relationships with sympathovagal balance, carbohydrate regulation, cortisol, and thyrotropin. J Clin Endocrinol Metab. 2004;89:5762-5771. doi:10. 1210/jc.2004-1003

29. Esposito K, Ciotola M, Schisano B, et al. Oxidative stress in the metabolic syndrome. $J$ Endocrinol Invest. 2006;29:791-795. doi:10.1007/BF03347372

30. Pihl E, Zilmer K, Kullisaar T, Kairane C, Magi A, Zilmer M. Atherogenic inflammatory and oxidative stress markers in relation to overweight values in male former athletes. Int $J$ Obes (Lond). 2006;30:141-146. doi:10.1038/sj.ijo.0803068

31. Jung SW, Lee KJ, Lee JH. Does weekend catch-up sleep affect high-sensitivity C-reactive protein levels among korean workers?: A cross-sectional study using KNHANES. J Occup Environ Med. 2019;61:e367-e73. doi:10.1097/JOM.0000000000001657

32. Dominguez LJ, Barbagallo M. The biology of the metabolic syndrome and aging. Curr Opin Clin Nutr Metab Care. 2016;19:5-11. doi:10.1097/MCO.0000000000000243

\section{Publish your work in this journal}

Diabetes, Metabolic Syndrome and Obesity: Targets and Therapy is an international, peer-reviewed open-access journal committed to the rapid publication of the latest laboratory and clinical findings in the fields of diabetes, metabolic syndrome and obesity research. Original research, review, case reports, hypothesis formation, expert opinion and commentaries are all considered for publication. The manuscript management system is completely online and includes a very quick and fair peer-review system, which is all easy to use. Visit http://www.dovepress.com/testimonials.php to read real quotes from published authors. 